Bull. Korean Math. Soc. 51 (2014), No. 4, pp. 1127-1133

http://dx.doi.org/10.4134/BKMS.2014.51.4.1127

\title{
NONADDITIVE STRONG COMMUTATIVITY PRESERVING DERIVATIONS AND ENDOMORPHISMS
}

\author{
Wei Zhang and Xiaowei Xu
}

\begin{abstract}
Let $S$ be a nonempty subset of a ring $R$. A map $f: R \rightarrow R$ is called strong commutativity preserving on $S$ if $[f(x), f(y)]=[x, y]$ for all $x, y \in S$, where the symbol $[x, y]$ denotes $x y-y x$. Bell and Daif proved that if a derivation $D$ of a semiprime ring $R$ is strong commutativity preserving on a nonzero right ideal $\rho$ of $R$, then $\rho \subseteq Z$, the center of $R$ Also they proved that if an endomorphism $T$ of a semiprime ring $R$ is strong commutativity preserving on a nonzero two-sided ideal $I$ of $R$ and not identity on the ideal $I \cup T^{-1}(I)$, then $R$ contains a nonzero central ideal. This short note shows that the conclusions of Bell and Daif are also true without the additivity of the derivation $D$ and the endomorphism $T$.
\end{abstract}

In this paper let $R$ always denote an associated ring with the center $Z$. For a semiprime ring $R$ denote by $C$ and $U$ its extended centroid and maximal right ring of quotients respectively (see [2] for details).

Recall that for a nonempty subset $S$ of a ring $R$, a map $f: R \rightarrow R$ is called strong commutativity preserving (scp) on $S$ if $[f(x), f(y)]=[x, y]$ for all $x, y \in S$, where the symbol $[x, y]$ denotes $x y-y x$. The concept of strong commutativity preserving maps was first introduced by Bell and Mason [4].

Bell and Daif [3] proved that if a derivation $D$ of a semiprime $\operatorname{ring} R$ is strong commutativity preserving on a nonzero right ideal $\rho$ of $R$, then $\rho \subseteq Z$, the center of $R$ (see also [11, 12] due to Liu et al. and [13] by Ma et al. for the results on generalized derivations). In [3] Bell and Daif also obtained that if an endomorphism $T$ of a semiprime ring $R$ is strong commutativity preserving on a nonzero two-sided ideal $I$ of $R$ and not identity on the ideal $I \cup T^{-1}(I)$, then $R$ contains a nonzero central ideal.

Brešar and Miers [5] proved that for a semiprime ring $R$ an additive map $f: R \rightarrow R$ being strong commutativity preserving on $R$ must have the form $f(x)=\lambda x+\xi(x)$, where $\lambda \in C, \lambda^{2}=1$, and $\xi$ is an additive map of $R$ into $C$.

Received September 5, 2013; Revised January 27, 2014

2010 Mathematics Subject Classification. 16W25, 16N60.

Key words and phrases. semiprime ring, prime ring, strong commutativity preserving map.

The work is supported by the NNSF of China (No. 11371165, No. 11101175 and No. 11071097), 211 Project, 985 Project and the Basic Foundation for Science Research from Jilin University. 
Lin and Liu [10] characterized the strong commutativity preserving additive maps on a noncentral Lie ideal of a prime ring.

The notion of strong commutativity preserving maps has been extended from different directions.

Deng and Ashraf [7] proved that a 2-torsion free prime ring $R$ is commutative if there is a non-identity endomorphism $T$ of $R$ such that $[T(x), T(y)]-[x, y] \in Z$ for all $x, y$ in some essential right ideal of $R$.

De Filippis and Scudo [6] introduced the concept of sep. According to the paper [6] a pair of maps $F$ and $G$ from a ring $R$ into itself is called mutually strong Engel condition preserving (sep) on $S(\subseteq R)$ if $[G(x), F(y)]_{h}=[x, y]_{h}$ for all $x, y \in S$, where the symbol $[x, y]_{h}$ is defined by the inductive procedure $[x, y]_{h}=\left[[x, y]_{h-1}, y\right]$. In $[6]$ both the pair of generalized derivations of a prime ring $R$ being sep on some subset of $R$ and the pair of derivations of a semiprime ring $R$ being sep on a Lie ideal of $R$ were explored.

Some maps similar to the map being scp are also explored. For example Ali and Huang [1] studied the derivation $d$ of a 2-torsion free semiprime ring $R$ satisfying $d(x) d(y)+d(y) d(x)=x y+y x$ or $[d(x), d(y)]=-[x, y]$ for all $x, y \in I$ (an ideal of $R$ ).

Recently nonlinear maps being scp on some subsets of a ring are focused and many valued conclusions are obtained.

Qi and Hou [14] considered the nonadditive strong commutativity preserving map and proved that for a unital prime $\operatorname{ring} R$ containing a nontrivial idempotent, a surjective map $f: R \rightarrow R$ is strong commutativity preserving on $R$ if and only if $f$ has the form $f(x)=\lambda x+\xi(x)$, where $\lambda \in\{-1,1\}$ and $\xi$ is an additive map of $R$ into $C$.

Liau, Huang and Liu [9] proved that for a prime ring $R$ of characteristic not 2 , with involution, with center $Z$ and with skew elements $K$, under a mild assumption, a map $f: K \rightarrow R$ is scp on $K$ if and only if $f(x)=x+\mu(x)$ for all $x \in K$ or $f(x)=-x+\mu(x)$ for all $x \in K$, where $\mu: K \rightarrow Z$ is a map.

Lee and Wong [8] characterized an arbitrary map $f$ such that $[f(x), f(y)]-$ $[x, y] \in Z$ for all $x, y \in S$, where $S$ is either a noncentral Lie ideal of a prime ring or a semiprime ring, extending many results in the literatures listed above perfectly.

It is a natural problem whether or not we can extend the conclusions by Lee and Wong [8] to the two-sided ideal (even the one-sided ideal) of a semiprime ring. But to our knowledge we could not reach a complete result. In this short note we only deal with a special case and prove that the conclusions of Bell and Daif [3] stated above are also true without the additivity of the derivation $D$ and the endomorphism $T$. We hope the results in this note can be extended to an arbitrary map.

In order to prove the main conclusions we first list some lemmas. We will cite Bell and Daif [3, Lemma 1(a)] frequently. So we state this result as Lemma 1. 
Lemma 1 (Bell and Daif). If $R$ is a semiprime ring, the center of a nonzero one-sided ideal is contained in the center of $R$; in particular, any commutative one-sided ideal is contained in the center of $R$.

Lemma 2 is implied by [2, Theorem 2.3.9 and Lemma 2.3.10].

Lemma 2. Let $R$ be a semiprime ring with a nonempty subset $S$. Then there exists an idempotent $\varepsilon \in C$ such that $\varepsilon s=s$ for all $s \in S$ and $\varepsilon T=0$, where $T=\{t \in R \mid S R t=0\}$.

Lemma 3 is a special case of Lemma 2.

Lemma 3. Let $R$ be a semiprime ring with a nonempty subset $S$ and a nonzero right ideal $\rho$. Then there exists an idempotent $\varepsilon \in C$ such that $\varepsilon s=s$ for all $s \in S \rho$ and $\varepsilon a \in C$ for $a \in R$ satisfying $S \rho R[a, \rho]=0$.

Proof. By Lemma 2 there exists an idempotent $\varepsilon \in C$ such that $\varepsilon s=s$ for all $s \in S \rho$ and $\varepsilon T=0$, where $T=\{t \in R \mid S \rho R t=0\}$. Particularly,

$$
[\varepsilon a, x]=0
$$

for all $x \in \rho$ since $S \rho R[a, \rho]=0$. Replacing $x$ by $x r$ in (1), where $x \in \rho$ and $r \in R$, then $x[\varepsilon a, r]=0$ by (1). That is $\rho[\varepsilon a, R]=0$. Moreover, $S \rho R[\varepsilon a, R]=0$. So by Lemma $2[\varepsilon a, R]=0$, which completes the proof.

We also need the following two lemmas.

Lemma 4. Let $R$ be a semiprime ring with $a \in R$. Then $a R a \subseteq Z$ if and only if $a \in Z$ and $a x \in Z$ for all $x \in R$.

Proof. We will only deal with the necessity. Firstly we get

$$
0=[a x a, a x]=a x[a, a x]=a x a[a, x]
$$

for all $x \in R$. Linearizing $x$ in (2) we obtain

$$
a x a[a, y]=-a y a[a, x]
$$

for all $x, y \in R$. Then by (2) and (3) for all $x, y \in R$

$$
a x a[a, y] \operatorname{Rax} a[a, y]=-a x a[a, y] \operatorname{Ray} a[a, x]=-a y a[a, y] \operatorname{Rax} a[a, x]=0,
$$

which means $a x a[a, y]=0$. Moreover $a[a, x] R a[a, x]=0$ which implies $a[a, x]=$ 0 for all $x \in R$. Then $a y[a, x]=a[a, y x]=0$ for all $x, y \in R$. So $a \in Z$ from $[a, x] R[a, x]=0$ for all $x \in R$. Furthermore $[a x, a y]=[a x a, y]=0$ for all $x, y \in R$. Thus $a x \in Z$ for all $x \in R$ by Lemma 1 .

Lemma 5. Let $R$ be a semiprime ring with a right ideal $\rho$. Then $\rho[\rho, \rho] \subseteq Z$ if and only if $\rho \subseteq Z$.

Proof. We only deal with the necessity. Assume $\rho[\rho, \rho] \subseteq Z$. We get

$$
[x, y] R[x, y] \subseteq Z
$$


which means $[x, y] \in Z$ for all $x, y \in \rho$ by Lemma 4 . Then $[y, z]^{2}=[y[y, z], z]=$ 0 which means $[y, z]=0$ for all $y, z \in \rho$, since $[y, z] \in Z$. So $\rho \subseteq Z$ by Lemma 1.

The proof of [3, Theorem 1] implies Lemma 6.

Lemma 6. Let $R$ be a semiprime ring with a nonzero right ideal $\rho$ and a map $\delta: R \rightarrow R$ such that $\delta(x y)=\delta(x) y+x \delta(y)$ for all $x, y \in R$. If $\delta$ is scp on $\rho$, then $\delta(x) \rho R[\delta(x), r]=0$ for all $x \in \rho$ and $r \in R$.

Now we will show that the conclusion of Bell and Daif [3, Theorem 1] is also true for nonadditive derivations.

Theorem 7. Let $R$ be a semiprime ring with a nonzero right ideal $\rho$ and a map $\delta: R \rightarrow R$ such that $\delta(x y)=\delta(x) y+x \delta(y)$ for all $x, y \in R$. If $\delta$ is scp on $\rho$, then $\rho \subseteq Z(R)$.

Proof. By Lemma 6, $[\delta(x), y] \rho R[\delta(x), y] \rho=0$ for all $x, y \in \rho$, which means $[\delta(\rho), \rho] \rho=0$. Computing $[\delta(x y), \delta(z)]=[x y, z]$ we deduce that for all $x, y, z \in$ $\rho$

$$
\delta(x)[y, \delta(z)]+[x, \delta(z)] \delta(y)=0 .
$$

Replacing $x$ by $t x$ in (4) and then applying $(4)$ and $[\delta(\rho), \rho] \rho=0$ we obtain $\delta(t) x[y, \delta(z)]=0$ for all $x, y, z, t \in \rho$. That is

$$
\delta(\rho) \rho R[\delta(\rho), \rho]=0 .
$$

Let $\mathcal{P}=\left\{P_{\alpha} \mid \alpha \in \Lambda\right\}$ be a family of prime ideals of $R$ such that $\cap P_{\alpha}=0$. Then for each $P \in \mathcal{P}$ either $\delta(\rho) \rho \subseteq P$ or $[\delta(\rho), \rho] \subseteq P$. If $[\delta(\rho), \rho] \subseteq P$, then for all $x, y, z \in \rho$

$$
y[x, z]=[\delta(x), y \delta(z)]-[\delta(x), y] \delta(z) \in P .
$$

So we always have $\delta(\rho) \rho R[\rho, \rho] \subseteq P$ for any $P \in \mathcal{P}$. That is $\delta(\rho) \rho R[\rho, \rho]=0$. Then by Lemma 3 there exists an idempotent $\varepsilon \in C$ such that $\varepsilon x=x$ for all $x \in \delta(\rho) \rho$ and $\varepsilon \rho \subseteq C$. Also by (5) and Lemma 3 we have $\varepsilon \delta(\rho) \subseteq C$. Then for all $x, y, z \in \rho$

$$
\begin{aligned}
& \delta(x)[y, \delta(z)]=\delta(x) y \delta(z)-\delta(x) \delta(z) y=(\varepsilon \delta(x) y) \delta(z)-\delta(x)(\varepsilon \delta(z) y) \\
= & \delta(x)(\varepsilon y) \delta(z)-\delta(x) \delta(z)(\varepsilon y)=\delta(x) \delta(z)(\varepsilon y)-\delta(x) \delta(z)(\varepsilon y)=0 .
\end{aligned}
$$

Review (4) then for all $x, y, z \in \rho$

$$
x \delta(z) \delta(y)=\delta(z) x \delta(y)=(\varepsilon \delta(z) x) \delta(y)=\varepsilon \delta(z)(\varepsilon x) \varepsilon \delta(y) \in C .
$$

Hence $x[y, z]=x[\delta(y), \delta(z)] \in C$ for all $x, y, z \in \rho$. Thus by Lemma $5 \rho \subseteq$ $Z(R)$.

Now we will show that the result of Bell and Daif [3, Theorem 3] for nonadditive endomorphism is also true. Firstly we give an equivalent condition under which a nonadditive endomorphism is strong commutativity preserving on a nonzero ideal of a semiprime ring. 
Theorem 8. Let $R$ be a semiprime ring with extended centroid $C$ and a nonzero ideal $I$. Let $\pi: I \rightarrow R$ be a map satisfying $\pi(x y)=\pi(x) \pi(y)$ for all $x, y \in I$. Then $\pi$ is scp on $I$ if and only if there exist two maps $\zeta: I \rightarrow \varepsilon C$ and $\tau: I \rightarrow(1-\varepsilon) R$ for some idempotent $\varepsilon \in C$ such that $\varepsilon z=z$ and $\pi(z)=z+\zeta(z)+\tau(z)$ for all $z \in I, \zeta(I) I \subseteq Z(R)$, and $[\tau(x), \tau(y)]=0$ for all $x, y \in I$.

Proof. The sufficiency can be seen from

$$
\begin{aligned}
{[\pi(x), \pi(y)] } & =[x+\zeta(x)+\tau(x), y+\zeta(y)+\tau(y)] \\
& =[\varepsilon x+(1-\varepsilon) \tau(x), \varepsilon y+(1-\varepsilon) \tau(y)] \\
& =[\varepsilon x, \varepsilon y]+[(1-\varepsilon) \tau(x),(1-\varepsilon) \tau(y)] \\
& =[x, y]+[\tau(x), \tau(y)]=[x, y]
\end{aligned}
$$

for all $x, y \in I$, since $\zeta(I) \subseteq C$. Now we will deal with the necessity. Through the following straightforward computation

$$
\begin{aligned}
x[y, z]+[x, z] y & =[x y, z]=[\pi(x y), \pi(z)] \\
& =[\pi(x) \pi(y), \pi(z)]=\pi(x)[y, z]+[x, z] \pi(y),
\end{aligned}
$$

we have that for all $x, y, z \in I$,

$$
(\pi(x)-x)[y, z]+[x, z](\pi(y)-y)=0 .
$$

Put $z=x$ in $(6)$ then $(\pi(x)-x)[y, x]=0$ holds for all $x, y \in I$. Moreover $(\pi(x)-x) y r[s, x]=(\pi(x)-x)[y r s, x]=0$ for all $x, y \in I$ and $r, s \in R$. Hence $[\pi(x)-x, x] I R[\pi(x)-x, x] I=0$ for all $x \in I$ which means $[\pi(x), x] I=0$ for all $x \in I$ since $R$ is semiprime. Particularly $[\pi(x), x]=0$ for all $x \in I$ from $[\pi(x), x] R[\pi(x), x]=0$. Replacing $z$ by $z(\pi(y)-y)$ in (6) and applying $[\pi(y), y]=0$ we get that for all $x, y, z \in I$

(7) $(\pi(x)-x)[y, z](\pi(y)-y)+[x, z](\pi(y)-y)^{2}+z[x, \pi(y)-y](\pi(y)-y)=0$.

Multiplying the two sides of (6) with $\pi(y)-y$ from the right hand side and then comparing with (7) we obtain that $z[x, \pi(y)-y](\pi(y)-y)=0$ for all $x, y, z \in I$. Furthermore for all $x, y, z \in I$ and $r, s \in R$

$$
z[r, \pi(y)-y] s x(\pi(y)-y)=z[r s x, \pi(y)-y](\pi(y)-y)=0 .
$$

Then $x[r, \pi(y)-y] R x[r, \pi(y)-y]=0$ for all $x, y \in I$ and $r \in R$. So $I[R, \pi(y)-$ $y]=0$ for all $y \in I$. Then $I R[R, T]=0$ where $T=\{\pi(y)-y \mid y \in I\}$. Hence by Lemma 2 there exists an idempotent $\varepsilon \in C$ such that $\varepsilon x=x$ for all $x \in I$ and $\zeta(y)=\varepsilon \pi(y)-\varepsilon y \in \varepsilon C$ for all $y \in I$. Thus $\pi(y)=y+\zeta(y)+\tau(y)$ for all $y \in I$ where $\tau(y)=(1-\varepsilon) \pi(y)$. The identity $\pi(x y)=\pi(x) \pi(y)$ implies the identity $\tau(x y)=\tau(x) \tau(y)$ for all $x, y \in I$, since $\tau(y)=(1-\varepsilon) \pi(y)$ for all $y \in I$. We also deduce $[\tau(x), \tau(y)]=0$ for all $x, y \in I$ from

$$
[\tau(x), \tau(y)]=(1-\varepsilon)[\pi(x), \pi(y)]=(1-\varepsilon)[x, y]=(1-\varepsilon) \varepsilon[x, y]=0 .
$$


At last we will prove $\zeta(I) I \subseteq Z(R)$. We can obtain that

$$
\zeta(x) y=(\pi(x)-x-\tau(x)) y=(\pi(x)-x) y
$$

for all $x, y \in I$, since $\tau(x) y=(1-\varepsilon) \pi(x)(\varepsilon y)=0$. Then for all $x, y \in I$, we get

$$
\begin{aligned}
x y+\zeta(x y)+\tau(x y) & =\pi(x y)=\pi(x) \pi(y) \\
& =x y+\zeta(x) y+\zeta(y) x+\zeta(x) \zeta(y)+\tau(x) \tau(y),
\end{aligned}
$$

since $\tau(x) y=0$ and $\tau(x) \zeta(y)=0$ for all $x, y \in I$. That is

$$
\zeta(x) y+\zeta(y) x+\zeta(x) \zeta(y)-\zeta(x y)=0
$$

for all $x, y \in I$. Hence $[\zeta(x) x, y]=[x, \zeta(x) y]=0$ for all $x, y \in I$, since $\zeta(I) \subseteq C$. Moreover $[\zeta(x) x, R] I=0$ for all $x \in I$. Particularly $[\zeta(x) x, R] R[\zeta(x) x, R]=0$ for all $x \in I$ which means $\zeta(x) x \in C$. Then we obtain that $\zeta(x)^{2} y \in C$ for all $x, y \in I$ through multiplying (8) by $\zeta(x)$ and using $\zeta(x) x \in C$. Hence $\zeta(x) y \in C$ for all $x, y \in I$ from $[\zeta(x) y, R] R[\zeta(x) y, R]=0$. At last noting that $\zeta(I) I \subseteq R$, we obtain that $\zeta(I) I \subseteq Z(R)$.

The conclusion by Bell and Daif is also true without the additivity of the endomorphism.

Corollary 9. Let $R$ be a semiprime ring with a nonzero ideal $I$ and a map $\pi$ : $I \rightarrow R$ satisfying $\pi(x y)=\pi(x) \pi(y)$ for all $x, y \in I$. If $\pi$ is strong commutativity preserving on $I$ and not the identity on $\pi^{-1}(I) \cap I$, then $R$ contains a nonzero central ideal.

Proof. By Theorem $8, \pi(x)=x+\zeta(x)+\tau(x)$ for all $x \in I$ and $\zeta(I) I \subseteq Z(R)$. Suppose $\zeta(I) I=0$. Let $x \in \pi^{-1}(I) \cap I$ with $\pi(x) \neq x$. Then $y=\pi(x) \in I$ for some $y \in I$. Hence $y=x+\zeta(x)+\tau(x)$ and then $(y-x) I=0$. So $y=x$ and $\pi(x)=x$, a contradiction. Consequently, $\zeta(I) I$ is a nonzero central ideal of $R$.

The following example shows that we could not get rid of the assumption that $\pi$ is not the identity on $\pi^{-1}(I) \cap I$ in Corollary 9 .

Example 1. Let $\mathcal{R}=\mathbb{M}_{2}(F) \times \mathbb{M}_{2}(F)$ where $\mathbb{M}_{2}(F)$ is the ring of all $2 \times 2$ matrices over a field $F$. Then $R$ contains no nonzero central ideals and $\pi: \mathcal{I} \rightarrow$ $\mathcal{R}$ satisfying $\pi(A, 0)=\left(A, \operatorname{det}(A) E_{11}\right)$ is a strong commutativity preserving map on $\mathcal{I}$, where $\mathcal{I}=\left\{(A, 0) \mid A \in \mathbb{M}_{2}(F)\right\}$ is a nonzero ideal of $\mathcal{R}$ and $E_{11}$ is the $2 \times 2$ matrix unit with $(1,1)$-entry being equal to 1 .

Acknowledgement. The authors would like to express their sincere thanks to the referee for his many good suggestions including grammar correcting, adding new references, and simplifying the proof of Corollary 9. The authors acknowledge that the current form of the proof of Corollary 9 is due to the referee completely for it is clearer than its previous form. 


\section{References}

[1] S. Ali and S. Huang, On derivations in semiprime rings, Algebr. Represent. Theory 15 (2012), no. 6, 1023-1033.

[2] K. T. Beidar, W. S. Martindale, and A. V. Mikhalev, Rings with Generalized Identities, Pure and Applied Mathematics 196, Marcel Dekker, New York, 1996.

[3] H. E. Bell and M. N. Daif, On commutativity and strong commutativity preserving maps, Canad. Math. Bull. 37 (1994), no. 4, 443-447.

[4] H. E. Bell and G. Mason, On derivations in near rings and rings, Math. J. Okayama Univ. 34 (1992), 135-144.

[5] M. Brešar, and C. R. Miers, Strong commutativity preserving maps of semiprime rings, Canad. Math. Bull. 37 (1994), no. 4, 457-460.

[6] V. De Filippis and G. Scudo, Strong commutativity and Engel condition preserving maps in prime and semiprime rings, Linear Multilinear Algebra 61 (2013), 917-938.

[7] Q. Deng and M. Ashraf, On strong commutativity preserving mappings, Results Math. 30 (1996), no. 3-4, 259-263.

[8] T. K. Lee and T. L. Wong, Nonadditive strong commutativity preserving maps, Comm. Algebra 40 (2012), no. 6, 2213-2218.

[9] P. K. Liau, W. L. Huang, and C. K. Liu, Nonlinear strong commutativity preserving maps on skew elements of prime rings with involution, Linear Algebra Appl. 436 (2012), no. 9, 3099-3108.

[10] J. S. Lin and C. K. Liu, Strong commutativity preserving maps on Lie ideals, Linear Algebra Appl. 428 (2008), no. 7, 1601-1609.

[11] C. K. Liu, Strong commutativity preserving generalized derivations on right ideals, Monatsh. Math. 166 (2012), no. 3-4, 453-465.

[12] C. K. Liu and P. K. Liau, Strong commutativity preserving generalized derivations on Lie ideals, Linear Multilinear Algebra, 59 (2011), no. 8, 905-915.

[13] J. Ma, X. W. Xu, and F. W. Niu, Strong commutativity-preserving generalized derivations on semiprime rings, Acta Math. Sin. (Engl. Ser.) 24 (2008), no. 11, 1835-1842.

[14] X. F. Qi and J. C. Hou, Nonlinear strong commutativity preserving maps on prime rings, Comm. Algebra 38 (2010), no. 8, 2790-2796.

WEI ZHANG

College of Mathematics

JILIN UNIVERSITY

Changchun 130012, P. R. China

E-mail address: cherish-bunny@qq.com

XiaOWEI Xu

College of Mathematics

JiLIN UNIVERSITY

Changlhun 130012, P. R. China

E-mail address: xuxw@jlu.edu.cn 